\title{
Towards Self-Managing Systems inspired by Economic Organizations
}

Arnautovic, E., Vallée, M., Mulvenna, M., Baumgarten, M., Hadjiantonis, AM., Rehm, S-V., Müthel, M., Karyotis, V., Papavassiliou, S., \& Stathis, K. (2010). Towards Self-Managing Systems inspired by Economic Organizations. In IEEE Conference on Systems, Man and Cybernetics (SMC-2010) (pp. 888-895). IEEE. https://doi.org/10.1109/ICSMC.2010.5641875

Link to publication record in Ulster University Research Portal

Published in:

IEEE Conference on Systems, Man and Cybernetics (SMC-2010)

Publication Status:

Published (in print/issue): 01/01/2010

DOI:

10.1109/ICSMC.2010.5641875

\section{Document Version}

Author Accepted version

\section{General rights}

Copyright for the publications made accessible via Ulster University's Research Portal is retained by the author(s) and / or other copyright owners and it is a condition of accessing these publications that users recognise and abide by the legal requirements associated with these rights.

\section{Take down policy}

The Research Portal is Ulster University's institutional repository that provides access to Ulster's research outputs. Every effort has been made to ensure that content in the Research Portal does not infringe any person's rights, or applicable UK laws. If you discover content in the Research Portal that you believe breaches copyright or violates any law, please contact pure-support@ulster.ac.uk. 


\section{Towards Self-Managing Systems inspired by Economic Organizations}

\author{
Edin Arnautovic, Mathieu Vallée \\ Vienna University of Technology \\ Vienna, Austria \\ \{arnautovic, vallee\}@ict.tuwien.ac.at
Maurice Mulvenna, Matthias Baumgarten University of Ulster
Jordanstown, Newtownabbey, UK
\{m.baumgarten, md.mulvenna\}@ulster.ac.uk \\ Antonis M. Hadjiantonis \\ University of Cyprus, KIOS Research Center \\ Nicosia, Cyprus \\ antonish@ucy.ac.cy
}

\author{
Sven-Volker Rehm, Miriam Müthel \\ WHU - Otto Beisheim School of Management \\ Vallendar, Germany \\ $\{$ sven-volker.rehm, miriam.muethel $\} @$ whu.edu \\ Vasileios Karyotis, Symeon Papavassiliou \\ National Technical University \\ Athens, Greece \\ vassilis@netmode.ntua.gr, papavass@mail.ntua.gr
}

Abstract-Today's self-managing systems would ideally be able to adapt themselves (their internal structure or behavior), as well as to autonomously participate in larger, self-organizing systems. Analogously, the enterpries or other socio-economic systems autonomously manage themselves - they make decisions on how to adapt their structure and behavior, and how to organize with other entities in the environment. To connect internal selfadaptive with external self-organizational behavior, an enterprise is "aware" of itself and of its environment, and acts according to this awareness.

This position paper proposes to address the challenges of a complex distributed self-managing system by making entities in such a system able to adapt themselves similarly to how companies manage in socio-economic systems. To enable the knowledge transfer between these two fields, the paper proposes to utilize symbolic models which will be used by self-managing systems for knowledge representation and reasoning. This will make such systems in a way also self-aware and enable both self-adaptive and self-organizing capabilities. The paper discusses research directions to make this approach possible.

Index Terms-self-managing systems, self-adaptive systems, self-organizing systems, autonomic computing, economic organizations

\section{INTRODUCTION}

Contemporary computing and communication systems become highly complex and heterogeneous, due to a vast amount of connected entities, varying context and structure, as well as dynamic interaction with human users and external services. Currently, such systems are not designed to adapt themselves to changing conditions or to optimize their resource usage. As a solution for these problems (in addition to e.g., hierarchical coordination and mathematical optimization), they should be selfmanaged. It is possible to identify two extreme approaches for self-managing systems, self-adaptation and self-organization. Figure 1 illustrates a possible kind of reconfiguration: creating a new relation between components of the system. This can be done either by self-adaption or by self-organization.

Self-adaptation: A promising approach for managing complex networked systems has been to transfer parts of the system management tasks from human operators to the systems themselves. "Self-adaptation puts the emphasis on individuals: the basic idea is of inherently autonomic components, which continuously monitor the environment and spontaneously trigger corrective actions whenever needed.'[1]. Such self-adaptive systems consist typically of the adaptable software, which provides actual system functionality, and an adaptation engine performing system management [2]. The basic idea here is to have the representation of architectural models of the system (typically based on an architecture description language) which are used during runtime by the adaptation engine to monitor the system, as well as to reason about possible system modifications [3]. Similar generic architecture of an autonomic system consisting of the autonomic manager, which controls the managed system, is presented in the context of autonomic computing initiative [4]. The focus of most research in this area was on how to design and develop sound architecture containing these two components and on the control loops to support it.

Self-organization: However, self-adaptiveness is not enough as a system's singular characteristic property. The systems should not be isolated but autonomously interact with other self-adaptive systems and jointly create desired behavior creating a larger, self-organizing system. "Self-organization puts the emphasis on groups and considers instead that adaptive behaviors can emerge from the interactions among simple components, without any central control (i.e. the so called swarm intelligence).’[1]. 


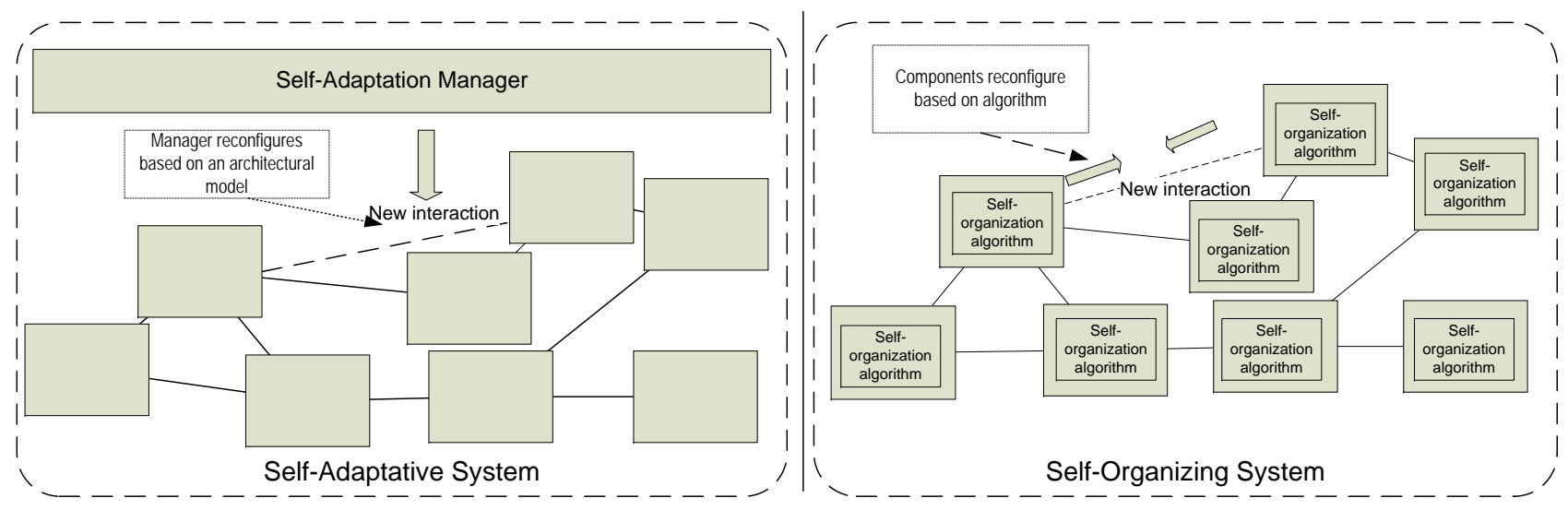

Fig. 1. Self-adaptive and self-organizing systems.

Analogously, the main goal of enterprises or other socioeconomic systems is to optimize the management of scarce resources and adapt themselves. They are aware of their own identity, capabilities and of the status of their current parameters (e.g., financial data or inventory). Based on this self-awareness, they make decisions how to act in a particular situation, how to interact with their environment, and how to coordinate information and actions on global and strategic levels with those on local and operative levels.

We consider it a realistic scenario that future complex systems would need to both adapt themselves (their behavior or internal structure), as well as to take part in larger, selforganizing systems. However, current self-adaptive systems concentrate on the internal view of the architecture of a single system, while self-organizing systems focus on the environment and procedural (algorithmic) issues. As a solution, to become self-managed, complex systems should be aware of what is happening to them and act according to this awareness. They should be self-aware.

We propose symbolic models inspired by socio-economic systems to integrate self-representation of the self-managing system with the representation of the systems's environment. These models consists of a network of concepts such as entities in the systems' environment, internal components, abstract concepts as rules, processes and laws, and the relationships between those concepts. They are opposite to numerical or geometrical models (however, they can influence each other). Symbolic models can e.g., be represented as an ontology and will be used for reasoning and will include an explicit representation of the 'self' of a self-managing system. For the self-organizational behavior, the symbolic models will also integrate the entities and characteristics of the environment. So, this work aims at integrating models, i.e. properties of the system's self, concepts and entities from the environment.

The remainder of this paper is organized as follows. First, we give some background about self-managing socioeconomic systems. After explaining the analogy of such systems to complex, computer based systems, we show how selfawareness can be used as a central point for integrating selfmanaging properties in such systems. This leads to symbolic models that should enable self-awareness within these systems. Finally, we present different research challenges for achieving that and give some possible research directions.

\section{BACKGRound In SElF-MANAGING SOCIO-ECONOMIC SYSTEMS}

Economic organizations are able both to self-adapt, e.g., by changing their internal structure or behavior, as well to participate in larger self-organizing structures (clans, value networks, etc.) [5]. As socio-economic systems they exhibit characteristics desired for the complex technical systems considered here. Because of this analogy, we envision the benefits of transferring relevant concepts and principles of socio-economic systems to technical, computer-based systems which should have comparable capabilities. In particular the following characteristics of socio-economic systems are significant:

- Their main task is to (self)manage and optimize usage of scarce resources, for the ultimate goals of sustaining their existence (social), creating value and profit (economic) and common wealth.

- They are self-adaptive, i.e. they operate and adapt themselves independently of any external controlling entity, exhibiting exciting abilities to react on external influences and to self-adapt flexibly (e.g. improvisation).

- They create self-organizing structures with an innate identity and specific characteristics, adopting distinct positions in the environment, and they learn to communicate and act within these structures and with the environment (relationship rules, contracts). These complex interactions with other entities and systems contribute to constant change and extension of those systems' structures and abilities.

Economic organizations perform these tasks by making decisions based strongly on knowledge that they have about their own current structure and properties, and about their environment. We postulate that the key feature of economic organizations concerning our problem is their awareness regarding their functioning as an economic actor, including both their internal organization and their external relations with 
other economic organization, as well as the mutual influence between the internal and external aspects.

We can illustrate the concepts related to self-awareness and related self-management in socio-economic systems using the example of a car manufacturing company within its value network (Figure 2). As depicted in the figure, various factors typically affect the operation of a company in its environment. For instance, the company is affected by other economic entities (e.g., customers, suppliers, competitors) and other social institutions and conditions (e.g., unions, laws and regulations). It needs to know about factors affecting it dynamically, such as, market trends, situations of co-operators, economic cycles, opportunities for innovation, etc. Finally, its operation depends on its internal functional structure, processes and resource constraints (e.g., production, purchase and sales departments, business processes internal and with external partners, personnel and resource cost). For a successful operation, the company needs to collect and interpret information about these factors, developing knowledge about strategic, tactic and operational status and options.

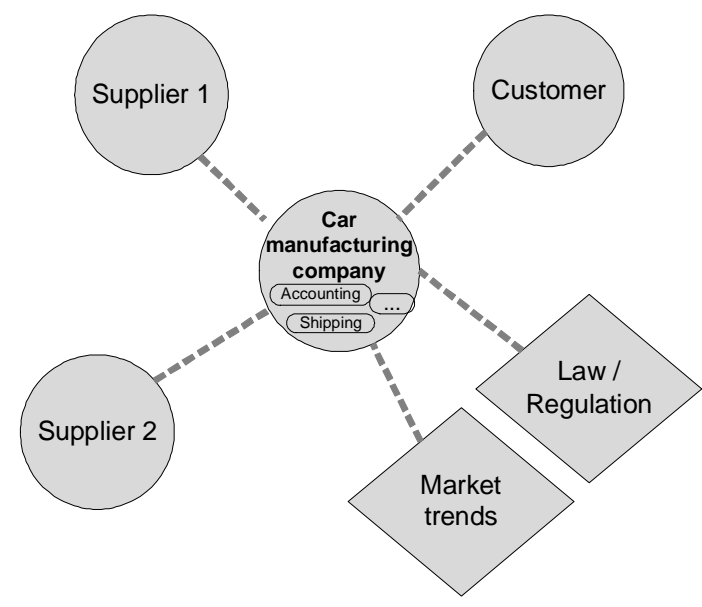

Fig. 2. Car manufacturing company as an economic, self-managing system.

Researchers have found that the economic organizations adapt their internal structure according to the properties of the environment [6]. In a more dynamic, uncertain environment, companies choose more organic, decentralized structure. In a more static, predictable environment companies adapt their structure to be more "mechanic" and centralized. They "consciously" identify possibilities for adaptation of their behavior and structure. In other words, we can say that the company (as a whole entity) needs to be "self-aware".

Aspects of self-awareness can be extended to the interoperation of companies within enterprise networks. Using approaches for Community Governance [7], even large-scale networked structures, comprising hundreds of partners, are able to synthesize context-dependent network structures by selforganization on basis of shared agreements, procedures and tools for knowledge exchange. Within enterprise networks, particular knowledge management tools and flexible, adaptable knowledge exchange frameworks reacting the inner structure and outer environment of a company have been proposed to enable a single network node to interoperate as an active agent within its network of partners.

In the example of the car manufacturing company a situation of resource scarcity might emerge, which entails a couple of considerations: It needs to investigate alternative transport means, securing future compliance to carbon emission rates (Law). For economic reasons, it needs to decide between hierarchical and market-type coordination with suppliers (Supplier 1, Supplier 2), and business partners. It also needs to develop a strategy for coping with changing boundary conditions, eventually leading to new relationship types, or to a change in the overall topography of the described value added network. It can even result in changes to the internal structure (regarding the organization of production).

\section{SElF-MANAGING TECHNiCAL Systems}

As an analogy to the socio-economic system discussed above, following example illustrates a self-organizing, selfadaptive technical system. We highlight on the one hand the functional analogy of entities, and on the other hand, the conceptual transfer from economic organizations to technical environments. We focus on self-representation using symbolic models and resulting self-awareness.

By designing self-managing complex systems it is important to identify what are the individual nodes (subsystems) which constitute a self-organizing system. For this paper we define a self-adaptive node as an element of a self-organizing system. Such nodes are internally self-adaptive where a more or less central entity controls the adaptation. Through their external interactions, these subsystems (nodes) participate in a larger, self-organizing system. However, such self-adaptive node can be very complex on its own and could fulfill its purpose even without participating within the larger, self-organizing system. In our running example, we define a large data center as one self-adaptive node.

Concerning the analogy to economic systems we see a single economic organization (e.g., a company as a legal entity) as the analogy to the self-adaptive node. Larger compositions of such economic organizations, which organize themselves (e.g., in value networks) to better pursue their interests leading to the emergent behavior and larger goal, are analogue to technical self-organizing systems.

The example shows a cloud computing provider (Figure 3 . A cloud is a large pool of easily usable and accessible virtualized resources (such as hardware, development platforms and/or services) which can be dynamically re-configured to adjust to a variable load and scale (adapted from [8]). This provider operates a complex self-adaptive data center which contains advanced infrastructure consisting of many hardware servers hosting different software (operating systems, virtualization frameworks, applications, etc.), fast network, power generators, air conditioners, etc. Many of the management tasks are not performed by humans but by the software component, the adaptation manager. The data center is (partially) 
self-managed - it is able to adapt itself to different conditions and to the new or evolving requirements.

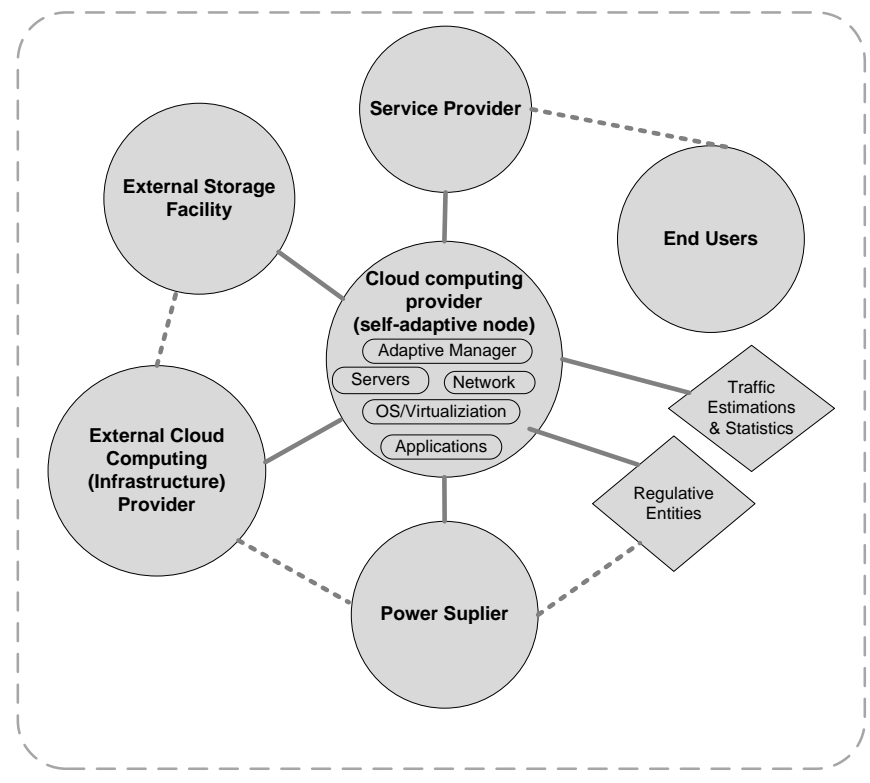

Fig. 3. Cloud computing provider as self-adaptive node in a self-managing complex technical system.

However, this data center is not isolated. It interacts intensively with its environment. For our example, we take the inspiration for such interactions from the Open Federated Cloud Computing Approach [9] where the data center automatically fulfills some of its additional computational or storage needs from other infrastructure providers in the cloud. Even if the cloud computing providers claim unlimited resources, this approach could offer them even larger flexibility. A further scenario is if this cloud computing provider is not public but operates an advanced, however, limited data center of one organization (e.g., university or a company). To optimize its costs-quality ratio the data center automatically and dynamically looks for other providers for its additional needs. Beside interactions with other similar organizations, it interacts also with its costumers - providers of business services on the top of the provided infrastructure (e.g., online shops or personal storage providers like DropBox 1 , which again interact with the end users. Other organizations such as the suppliers of electrical energy are also relevant. The cloud provider chooses the most appropriate energy supplier dynamically (e.g., the one which offers the lowest costs when the server load and energy consumption is the highest).

Being involved in such interactions for the purpose of optimizing their performance (e.g., in the terms of costs or energy consumption), the organizations establish a composite, self-organizing system.

\section{A. Self-awareness using integrated Symbolic Models}

As stated above, the "awareness" of the internal structure and properties, as well as of the environment in which it

\footnotetext{
${ }^{1}$ www.dropbox.com
}

operates is important for economic organizations to achieve (internal) self-adaptiveness and (external) self-organizing. In economic systems, such information is implicitly existing (e.g., as organigrams, diagrams, or parameters such as financial data or inventory) and is used by executive personnel to discuss, reason about or implement different decisions. To achieve the same awareness in a technical system and thus the same selfadaptive and self-organizing behavior, the system should also be able to represent and use this information, e.g., in a the form of models.

Such models have to be machine readable and understandable, and they provide the foundations for the (technical) creation of self-, and environmental-"awareness" for self-adaptive nodes. To do that, we propose these models to contain explicit representation of the "self". The "self" contains relevant knowledge about the particular self-adaptive node. This includes relevant context-dependent characteristics, properties and their current value and functions that such nodes can perform including their consequences. It also contains information about the communication in which this particular node can be involved or is currently participating.

A major challenge in self-organizing systems is the ability of a single node to maintain an accurate internal representation of relevant information about the environment in which it operates, and achieve this in the most efficient manner. The inability to achieve this hinders effective task planning and execution. Symbolic representation of the "self" and the resulting selfawareness will empower a self-adaptive node to examine and adapt its own behavior with consideration of the information from the external environment. In addition, the "self" will be related to the knowledge about the environment of such a node, for example about other nodes and their visible properties, as well as relevant external information such as temperature or geographic position. Thus, all knowledge that the self-adaptive node requires will be integrated into one symbolic model.

Figure 4 represents such symbolic model for the example above. We can think about the elements of this model as instances in a Unified Modeling Language (UML) model, or individuals in an ontology. The model contains the representation of the "self" of the Data Center as its central element with two important properties, currentStorageLoad and currentCPULoad. These properties are important for the reasoning about the behavior of the Data Center. For example, if the storageLoad is higher than some threshold, the data center (its self-adaptation manager) decides to outsource some of the data to the external storage provider (e.g., until its own storageLoad falls under the threshold). To be able to perform such outsourcing, the self-adaptation manager needs to "know" about the external storage facilities and how it is related to them. The relations in the model are represented by lines, e.g., the line connecting the Data Center and the External Storage Facility (labeled outsourceTo). The star (*) indicates that the Data Center is related to several external storage facilities. The relations to external entities in such symbolic model have their own properties such as the priority or the importance (as expressed by the line thickens in the figure). Representations of 


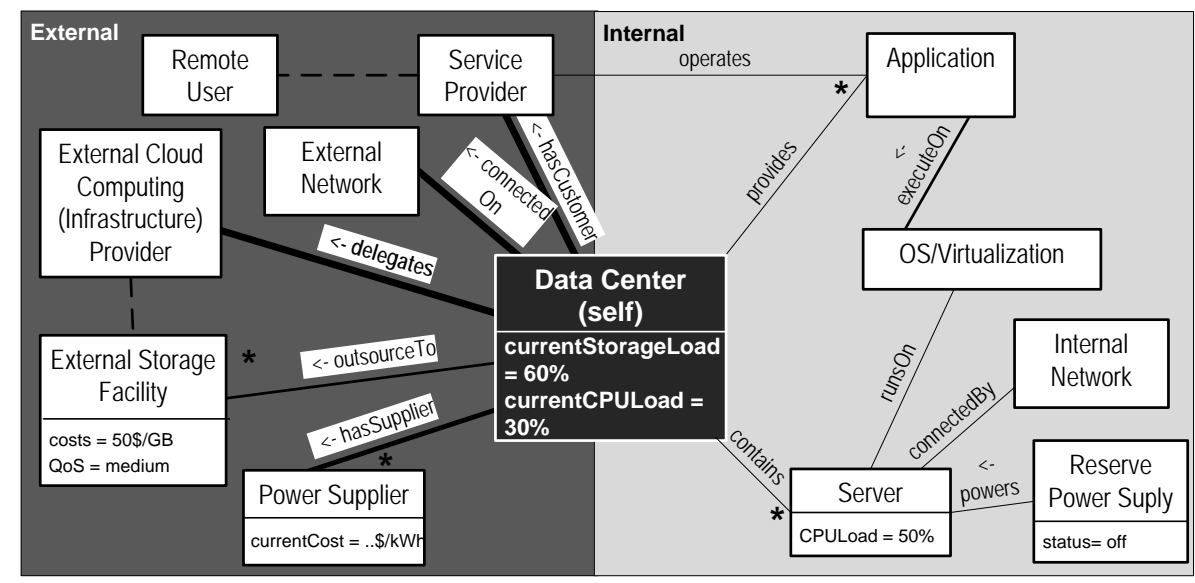

Fig. 4. Example of a symbolic model for a self-managing system.

each of these facilities includes also relevant parameters (e.g., costs and Quality of Service) which enable the Data Center to reason about when, of which data, or to which of the external storage provider to perform the outsourcing.

The Data Center also manages information about its internal structure (right in the figure). It contains many Servers which are connectedBy the internal network (to avoid the cluttering of the figure, not all possible relations are shown). Representation of a server contains e.g., information of its current CPU load. This information is used to adapt the internal behavior and structure of the data center as well as to calculate some parameters of the Data Center (e.g., currentCPULoad). So the system in the example becomes aware of its "social" and "external" views of the surrounding environment, in addition to being aware of itself and of its "internal" conditions.

Such symbolic models are to be utilized within the selfadaptation manager of the single node. Please note that this manager is responsible both for the adaptation of the node making the node self-adaptive, as well as for the interaction with other nodes for the integration into larger, self-organizing system. This is depicted in Figure 5. The self-adaptation manager of the node on the right in the figure contains and utilizes the integrated symbolic model making the node selfaware. Based on this, the node can (1) self-adapt, and (2) interact with external nodes executing self-organizing algorithm. Other systems (nodes) act also according to this algorithm. An self-organizing system emerges through this interaction and cooperation.

\section{Discussion and Research Challenges}

The vision stated above yields a number of research questions:

- The identification of relevant socio-economic concepts and principles: How to identify which concepts and principles in the management of companies are the most likely to yield interesting results in self-organizing architectures? What are the key properties to be found in companies that would also be relevant in self-managing systems?
- The transfer of relevant socio-economic concepts and principles: How to transfer these concepts and principles into a technical system? How to model the organization of the complex technical system, as well as the dynamic changes in this organization? How to relate the organization of the system with the internal organization (architecture) of nodes?

- The transfer of decision-making mechanisms: How to reproduce the typical decision-making mechanisms of a company into a technical system? In particular, how to adjust between various reasoning modes and granularity levels depending on dynamic conditions? How to manage the role of time in decision-making?

- The transfer of knowledge-monitoring mechanisms: How to reproduce the typical knowledge monitoring mechanisms necessary for a company to take the right decisions? In particular, how to properly manage the acquisition of implicit or tacit knowledge necessary for strategic decisions? Also, how to manage trust and reputation with regards to other entities in the system?

In this section, we give an overview on these questions and we sketch potential research directions.

\section{A. Identification of socio-economic concepts and principles}

In social and economic systems, sensations of selfawareness can be ascribed to "nodes" (persons, teams, formal or informal organizational structures etc.), generally associated with notions of autonomous behavior, self-dependency, intelligence, adaptiveness, and others. In order to learn from such phenomena and understanding, it is necessary to analyze existing theories about those systems, with the aim to conceptualize existing disciplinary knowledge. Transferring existing concepts from social sciences requires in a first step, to understand in which form the concept of self-awareness is existing in those theories and models, how it plays together with other concepts, and how it is implemented. In a second step this knowledge will be used to create symbolic models of this understanding which can be represented by self-adaptive, selforganizing systems. A couple of potentially promising theories 


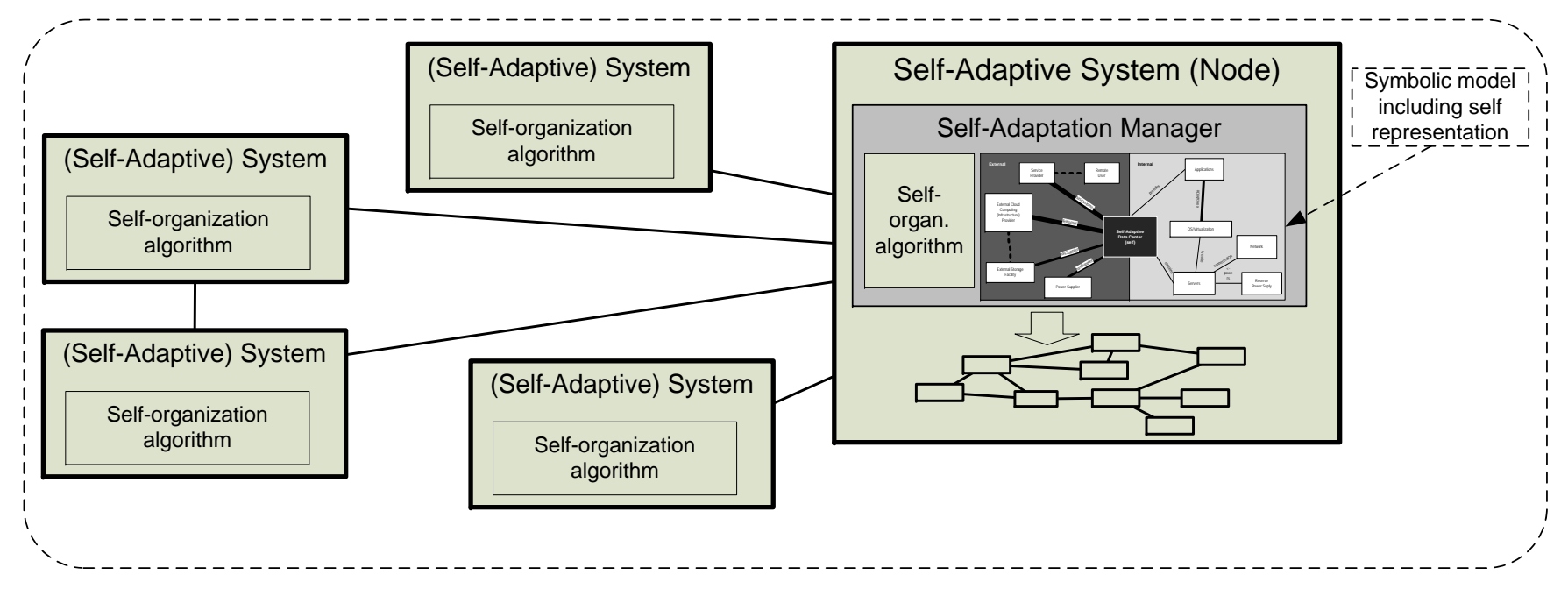

Fig. 5. Self-managing system.

exist, which could serve as guidelines for the engineering of self-adaptive, self-organizing systems.

The theory of structuration [10] deals with the idea of the duality of structure that allows relating between the levels of individual action and socio-institutional context, both being equally relevant within organizations. It is today used for empirical studies and provides itself a model containing aspects of structure, modality and interaction, in order to provide insights into motivation of individual action within a social context and the nature of social structures that are created virtually through social activity.

From an organizational behavior view, symbolic models exhibiting self-awareness and being able to reason can be compared to self-managed geographically dispersed teams, with knowledge residing at different locations. Self-managed teams do not have a vertical leader exerting authority and imposing task solutions. Instead, team members bring in their specific expertise and argue for or against a decision alternative. Such teams unfold their potential, when engaging in administrative (plans, procedures etc.) and expertise coordination (using taskdependent combination of individual and collective knowledge) [11].

The concept of identity is increasingly used in the social sciences describing how individuals and organizations differentiate themselves and emphasize their distinctiveness. It is also understood as a structural property of networks and organizations. Aspects are how the range of strong and weak contexts in modern organizations affects identification, or how identification is shaped and deployed [12].

In Management Science, in particular Knowledge Management theories point at the role of knowledge, its use within formal and informal structures, and its role as prerequisite for self-organization and adaptivity through emerging knowledge processes [13].

In the area of Business Engineering, aspects of "smart" and virtual networking as well as advancements in supporting organization through more open information and communi- cation systems have given rise to a plethora of conceptions for collaborative networks and ecosystems, e.g. Communities, Dynamic Virtual Organization, Dynamically Networked Organization, Extended Enterprise, Breeding Environment, Professional Virtual Community, etc. [14]. Their base line is the adaptable exploitation of knowledge sources and distribution of information and knowledge within adaptable organizational structures, whereas each conception argues with respect to three distinct organizational levels (person-, organization- or network-centric) for their specific context.

\section{B. Transfer of socio-economic concepts and principles}

Our intention to construct technical systems that orient themselves at socio-economic systems is a typical design problem of Information Systems (IS) research. The Design Science approach [15] in this respect promises to be particularly suited for transferring socio-economic concepts and principles to selforganizing, self-adaptive systems. Hevner et al. point at the two basic paradigms of research concerned with artificial systems, behavioral science and design science: "The behavioral science paradigm seeks to develop and verify theories that explain or predict human or organizational behavior. The designscience paradigm seeks to extend the boundaries of human and organizational capabilities by creating new and innovative artifacts.

In order to learn from socio-economic theories and to transfer the relevant concepts and principles, it is important to identify the most significant ones and to abstract from low-level details. We propose to represent these concepts and their relations using symbolic models. As stated above, such models consists of a network of concepts and their relations, enchained with additional semantic information. One way is to develop a Domain Specific Modeling Language [16] (e.g., in the form of a metamodel) identifying the most significant concepts for (self)representation in socio-economic systems, and use this language for models of the (self)representation in self-adaptive, self-organizing systems. Such models should 
integrate a representation of the self and a representation of the environment (other entities) in a consistent way. For example, models presented in the previous section could be designed based on such language. Since such model makes statements what exists in this domain, it can be considered an ontology [17]. If it is represented formally (e.g., using Web Ontology Language - OWL), it can be basis for ontology-based reasoning.

Work in this direction can be found in the field of multiagent systems, in which self-representation is a valuable tool for managing the interplay between local and global information. For instance, works on coalition formation [18] or on coordination involve an explicit representation of one's capabilities and dependencies, as well as the relation with capabilities and needs of other agents. Such representation can be inspired by socio-economic models [18].

\section{Decision-Making Mechanisms}

To reproduce behaviors similar to economic organizations, new decision-making solutions are required. In particular, adjusting between various reasoning modes and granularity levels depending on dynamic conditions should be tackled using an adaptive reasoning approach. In analogy to the definition given by Turner [19], we define adaptive reasoning as the ability of a reasoning mechanism to adapt its behavior in response to the changes in the underlying system (e.g. data, stakeholders, relations, interaction, etc.) as well as the goal of the reasoning mechanism itself. Here the latter is of particular interest, as it would allow for introspective reasoning where assumptions inferred on a micro-level could be further validated or, if proven false, contradicted. Although an important area in general information technology terms, generic reasoning algorithms are still scarce.

Within the literature, reasoning principles are categorized to be inductive, deductive or abductive. Deductive reasoning, being a top-down approach allows to (in-) validate a theory or condition, which can be used for the generation of vertical overlays where generalized knowledge can be modeled at various granular levels. Inductive reasoning on the other hand is a bottom-up approach and seeks a broader generalization that is based on specific observations, which is of particular interest for the discovery of relations between stakeholders. Finally, abductive reasoning attempts to infer logical explanations that are based on observations. This is of interest when generating assumptions and for the extraction of descriptive explanations for the benefit of better human-computer or automated computer-computer interactions. Incorporating and maintaining the results of such reasoning exercises is a significant research challenge that implies the use of temporal overlays in order to differentiate between past, current and future system conditions.

\section{Knowledge-monitoring, and trust and reputation mecha- nisms}

Collecting and monitoring knowledge is necessary for selfmanaging system to reason and take decisions. In particular, we see the monitoring of data streams as a particularly challenging issue. Another dimension is the management of implicit knowledge about other entities, such as trust and reputation.

Large-scale distributed systems generate an enormous amount of data that need to be analyzed. Thus, extracting highly descriptive and ultimately actionable knowledge patterns from such a "non-stopping stream of information" is of vital importance. Research in this area has gained in importance in recent years and a review of the state of the art in this area has been presented in [20].

In addition, a number of open research issues have been identified which need to be tackled before real-time, streambased knowledge monitoring can be facilitated successfully. Analyzing continuous data streams that theoretically embrace an infinite amount of knowledge requires memory independent analyzing techniques - a problem not tackled by most classical analytical methods. For this, methods have to be employed that condense, analyze and correlate data on the fly. Furthermore, the collection of streamed data, as well as their decomposition, is paramount for the success of analyzing methods. Solutions for decomposing a continuous data stream into individual chunks of interest depending on subsequent analytical methods are needed. They would allow for dynamic data selection that provides data reduction, quality improvements and data cleansing before any analytical processing. Subsequent services would only process data that is of relevance to their goal instead of wasting processing power on irrelevant data.

Finally, individual models created to represent and analyze any changes in the underlying system, data or business goals, need to change accordingly to provide continuous quality of service. This phenomenon has been referred to as concept drift [21] where a number of discrete models is analyzed in an effort to predict the change within the analyzed model without the need to analyze the data itself. Successful adaptation of such methodologies towards stream-based environment has the potential to reduce the burden for in depth data analysis [22].

Social and topological awareness can be exploited for improving the operation and response of a complex system to its environment and variations of user behavior patterns. However, it does not suffice alone for establishing proper interactions and services that can be trusted to perform as designed, mostly because intended misuse, as well as compositions of actions may lead to unpredictable system behavior. Establishing trust and managing reputation of peers in complex self-managing systems is not a simple one-iteration procedure, but rather requires reconsideration of several parameters involved. More specifically, it is necessary to establish a framework under which legitimate of a complex system will be able to negotiate and trust each other [23]. Characteristically, information flow/representation, peer communication and computational models employed need to be re-evaluated in a manner that takes into account the achieved levels of node self-awareness, while it performs trust evaluation in a distributed computational manner that does not disrupt the autonomic functions of the node while operating in a highly dynamic environment 
[24]. Trust and reputation mechanisms need to be reconsidered to suit symbolic models presented above. Algebraic trust models have been developed [25] and they can constitute a basis for developing more environmentally-aware reputation building mechanisms, which in turn can be utilized to secure and ensure proper information exchange.

\section{CONCLUSiOn}

In this position paper we discuss development of selfmanaging systems inspired by economic organizations. In particular, we address the integration of self-adaptive and selforganizing capabilities of self-managing systems. Assuming the analogy between socio-economic systems and technical systems, and their respective inherent or developed selfawareness, we aim at transferring the knowledge about representation and usage of self-awareness from socio-economic to self-managing systems. Using self-awareness as a core concept, such systems might be able to realize self-adaptiveness, as well as to take part in larger self-organizing systems. To realize this self-awareness technically, we propose symbolic models explicitly representing the "self" which are used for reasoning within such systems. We present several promising theories and research directions to make such models and resulting selfawareness feasible. We believe that integrating integrating selfadaptation and self-organization capabilities around symbolic models and self-awareness will improve the self-managing properties of complex computer based systems.

\section{REFERENCES}

[1] R. Quitadamo and F. Zambonelli, "Autonomic communication services: a new challenge for software agents," Autonomous Agents and MultiAgent Systems, vol. 17, no. 3, pp. 457-475, 2008.

[2] M. Salehie and L. Tahvildari, "Self-adaptive software: Landscape and research challenges," ACM Trans. Auton. Adapt. Syst., vol. 4, no. 2, pp. 1-42, 2009. [Online]. Available: http://dx.doi.org/10.1145/1516533. 1516538

[3] D. Garlan, S.-W. Cheng, A.-C. Huang, B. Schmerl, and P. Steenkiste, "Rainbow: Architecture-based self adaptation with reusable infrastructure," IEEE Computer, vol. 37, no. 10, 2004.

[4] J. O. Kephart and D. M. Chess, "The vision of autonomic computing," IEEE Computer, vol. 36, no. 1, pp. 41-50, 2003.

[5] O. E. Williamson, The Mechanisms of Governance. Oxford University Press, 1996

[6] G. R. Jones, Organizational theory, design, and change, 6th ed. Prentice Hall, 2009.

[7] S. Rehm, "Community governance - management concept for value networks of textile industry," in In Proceedings of the 4th International Conference on Economics and Management of Networks (EMNet 2009), 2009.

[8] L. M. Vaquero, L. Rodero-Merino, J. Caceres, and M. Lindner, "A break in the clouds: towards a cloud definition," SIGCOMM Comput. Commun. Rev., vol. 39, no. 1, pp. 50-55, 2009.
[9] B. Rochwerger, D. Breitgand, E. Levy, A. Galis, K. Nagin, I. M. Llorente, R. Montero, Y. Wolfsthal, E. Elmroth, J. Caceres, M. Ben-Yehuda, W. Emmerich, and F. Galan, "The RESERVOIR model and architecture for open federated cloud computing," IBM Jour. of Res. and Dev., vol. 53, no. 4, 2009. [Online]. Available: http://www.research.ibm.com/journal/rd/534/rochwerger.pdf

[10] A. Giddens, The constitution of society: Outline of the theory of structuration. Berkeley: University of California Press, 1984.

[11] F. Siebdrat, M. Hoegl, and H. Ernst, "The bright side of virtual collaboration: How teams can profit from dispersion," in Academy of Management Proceedings, 1-6, 2008.

[12] R. S. Burt, Brokerage and Closure: An Introduction to Social Capital. Oxford University Press, USA, 2005. [Online]. Available: http://www.amazon.com/exec/obidos/redirect?tag= citeulike07-20\&path=ASIN/0199249148

[13] I. Nonaka and H. Takeuchi, The Knowledge-Creating Company: How Japanese Companies Create the Dynamics of Innovation. Oxford University Press, USA, 1995. [Online]. Available: http://www.amazon. com/exec/obidos/redirect?tag=citeulike07-20\&path=ASIN/0195092694

[14] L. M. Camarinha-Matos and H. Afsarmanesh, "The emerging discipline of collaborative networks," in Virtual Enterprises and Collaborative Networks, IFIP 18th World Computer Congress, TC5 / WG5.5 - 5th Working Conference on Virtual Enterprises, L. M. Camarinha-Matos, Ed. Kluwer, 2004, pp. 3-16.

[15] A. R. Hevner, S. T. March, J. Park, and S. Ram, "Design science in information sys-tems research," MIS Quarterly, vol. 28, no. 1, pp. 75105, 2004.

[16] S. Kelly and J.-P. Tolvanen, Domain-Specific Modeling: Enabling Full Code Generation. Wiley-IEEE Computer Society Pr, 2008. [Online]. Available: http://www.worldcat.org/isbn/0470036664

[17] B. Chandrasekaran, J. R. Josephson, and V. R. Benjamins, "What are ontologies, and why do we need them?" IEEE Intelligent Systems, vol. 14 , no. 1 , pp. 20-26, 1999.

[18] J. S. Sichman, "DEPINT: Dependence-based coalition formation in an open multi-agent scenario," Journal of Artificial Societies and Social Simulation, vol. 1, no. 2, p. 3, 1998. [Online]. Available: http://www.soc.surrey.ac.uk/JASSS/1/2/3.html

[19] R. M. Turner, Adaptive Reasoning for Real-World Problems: A SchemaBased Approach. Hillsdale, NJ, USA: L. Erlbaum Associates Inc., 1994.

[20] M. M. Gaber, A. Zaslavsky, and S. Krishnaswamy, "Mining data streams: a review," ACM SIGMOD Record, vol. 34, no. 2, pp. 18-26, 2005. [Online]. Available: http://doi.acm.org/10.1145/1083784.1083789

[21] M. Black and R. Hickey, "Learning classification rules for telecom customer call data under concept drift," Soft Computing - A Fusion of Foundations, Methodologies and Applications, vol. 8, no. 2, pp. 102-108, 2003. [Online]. Available: http://dx.doi.org/10.1007/s00500-002-0250-2

[22] M. Baumgarten, K. Greer, M. D. Mulvenna, K. Curran, and C. D. Nugent, "Utilizing concept drift for pervasive supervision," in Proceedings of the Inter. Conf. on Software Engineering Research \& Practice (SERP 2008), 2008, pp. 688-694.

[23] H. Lutfiyya, M. Perry, and C. Yew, "Trust and autonomic systems," in Proc. of International MCETECH Conference on e-Technologies, 2008.

[24] T. Jiang and J. S. Baras, "Trust evaluation in anarchy: A case study on autonomous networks," in Proc. of 25th IEEE Conference on Computer Communications (INFOCOM), 2006.

[25] G. Theodorakopoulos and J. S. Baras, "On trust models and trust evaluation metrics for ad hoc networks," IEEE Journal on Selected Areas in Communications (JSAC), vol. 24, no. 2, pp. 318-328, 2006. 